\title{
An improved WENO-Z scheme for a multi-class Lighthill-Whitham-Richards traffic flow model
}

\author{
Pan Luo ${ }^{1, ~ a, ~ L i n g y a n ~ T a n g ~}{ }^{2, b}$ \\ ${ }^{1,2}$ College of Science, National University of Defense T echnology, Changsha 410073, China \\ alp2010cshd@163.com, ${ }^{b}$ tanglingyan@aliyun.com
}

Keywords: Multi-class model, WENO-Z, Central-upwind scheme, TVD Runge-Kutta.

Abstract. In this paper, we mainly propose a new semi-discrete central-upwind scheme to solve a MCLWR model based on fifth order improved WENO-Z reconstruction and semi-discrete central-upwind numerical fluxes. By introducing the new WENO-Z reconstruction, the order of accuracy of the schemes is improved, and preserve essential non-oscillatory property. The main advantage of this procedure is that the capacity of much better resolution at the smooth parts of the solution, while keeping the same numerical stability of the original WENO-Z at shocks and discontinuities. This property must be contrasted with classical upwind WENO schemes. The time integration we adopt is the third order TVD Runge-Kutta scheme. The effectiveness of our method is demonstrated in several numerical examples.

\section{Introduction}

The fluid mechanics of traffic flow models treat the traffic flow as compressible continuous medium consist of large amounts of vehicles, and investigate average behavior of vehicles cluster. The development of this model was derived from the LWR model [1]. The LWR model can describe a variety of real traffic phenomena such as shock formulation and the dispersion of the traffic jam, however the vehicles always derive at this equilibrium velocity, cannot reveal vehicles motion at the non-equilibrium. To overcome deficiencies of the LWR model, many kinds of multi-class LWR models were proposed, the model proposed by Wong and Wong [2] is researched much recently, this model can simulate the influence of vehicles with different velocity in congested and non-congested traffic regime, such as overtaking as well as slower vehicles would slow down the faster ones.

In numerical simulations, many efforts were done for MCLWR model, Zhang et al.[3] applied the weighted essentially non-oscillatory(WENO) scheme to the MCLWR model with heterogeneous drivers, and Chen et al.[4] adopted the fourth relax scheme, and Ngoduy and Liu [5] use the first order HLLE(Harten-van Leer-Lax and Einfeldt) scheme and so on.

In this paper, we research another high-resolution numerical method, which is semi-discrete central-upwind scheme [6] based on an improved WENO-Z reconstruction and strong stability-preserving Runge-Kutta method [7].

\section{The MCLWR Model}

In this section, we recall the MCLWR model [2] proposed by Wong and Wong, which was extended from the LWR model [1]. Let there be $M$ classes of road users with different speed choice behaviors in response to the same traffic density when traveling on a highway section, then apply the continuity equation for each different user classes. Let $\rho_{m}(x, t)$ and $u_{m}(x, t)$ denote the density and speed of user class $m$, respectively. The MCLWR model can be written in conservation form as

$$
U_{t}+F(U)_{x}=0 \text {, }
$$

where $U=\left[\rho_{1}, \rho_{2}, \ldots, \rho_{M}\right]^{\mathrm{T}}$ and $F(U)=\left[\rho_{1} u_{1}, \rho_{2} u_{2}, \ldots, \rho_{M} u_{M}\right]^{\mathrm{T}}$.Introducing the speed-density relationship presented by Wong and Wong

$$
u_{m}(x, t)=v_{m}(\rho), \forall m=1,2, \ldots, M
$$


where $\rho=\sum_{m=1}^{M} \rho_{m}$ is the total density. Assumed that the $m$ vehicular species are numbered from the slowest to the fastest, which is

$$
v_{1}(\rho)<v_{2}(\rho)<\mathrm{L}<v_{m}(\rho)
$$

According to Zhang et al. [8], $U$ satisfy

$$
U \in D, D=\left\{U \mid \rho_{m}>0, m=1,2, \ldots, M ; \sum_{m=1}^{M} \rho_{m}<\rho_{j a m}\right\}
$$

where $\rho_{\text {jam }}$ is the jam density, the eigenvalues $\left\{\lambda_{m}\right\}_{m=1}^{M}$ of the Jacobian $F_{U}$ satisfy

$$
v_{1}+\sum_{m=1}^{M} \frac{\partial v_{m}}{\partial \rho}<\lambda_{1}<v_{1}<\lambda_{2}<\mathrm{L}<v_{m-1}<\lambda_{m}<v_{m}<\mathrm{L}<v_{M-1}<\lambda_{M}<v_{M}
$$

\section{Numerical Schemes}

We apply the central-upwind scheme to solve the MCLWR system which is proposed by Kurganov et al. [6] for solving hyperbolic conservation laws. For simplicity, we consider the case of the uniform grid. The computational domain is discretized into a uniform mesh of $N$ grid points:

$$
x_{j}=j \Delta x ; \quad j=1,2, \ldots, J
$$

where $\Delta x$ is the uniform mesh size on the spatial axis.

Denote the cell-average in the cell $I_{j}=\left(x_{j-\frac{1}{2}}, x_{j+\frac{1}{2}}\right)$ by $\bar{u}_{j}$ :

$$
\bar{u}_{j}=\frac{1}{\Delta x} \int_{I_{j}} u(\xi) d \xi
$$

For the multi-class case with $M>2$, it's difficult to obtain eigenvalues of the system explicitly [8, 9]. Therefore, in this paper, the estimate of the local speed is based on a set of staggered inequalities of eigenvalues Eq. 5

$$
\begin{aligned}
& a_{j+\frac{1}{2}}^{-}=\min \left\{v_{1}+\left.\sum_{m=1}^{M} \rho_{m} \frac{\partial v_{m}}{\partial \rho}\right|_{U=U_{j+\frac{1}{2}}^{+}}, v_{1}+\left.\sum_{m=1}^{M} \rho_{m} \frac{\partial v_{m}}{\partial \rho}\right|_{U=U_{j+\frac{1}{2}}^{-}}, 0\right\} \\
& a_{j+\frac{1}{2}}^{+}=\max \left\{\left.v_{M}\right|_{U=U_{j+\frac{1}{2}}^{+}},\left.v_{M}\right|_{U=U_{j+\frac{1}{2}}^{-}}, 0\right\}
\end{aligned}
$$
[6]

Finally, our semi-discrete central upwind scheme can be written in the following conservation form

$$
\frac{d}{d t} U_{j}(t)=-\frac{H_{j+\frac{1}{2}}(t)-H_{j-\frac{1}{2}}(t)}{\Delta x}
$$

Here, the numerical fluxes $H_{j+\frac{1}{2}}$ are given by [10]

$$
H_{j+\frac{1}{2}}(t)=\frac{a_{j+\frac{1}{2}}^{+} F\left(U_{j+\frac{1}{2}}^{-}(t)\right)-a_{j+\frac{1}{2}}^{-} F\left(U_{j+\frac{1}{2}}^{+}(t)\right)}{a_{j+\frac{1}{2}}^{+}-a_{j+\frac{1}{2}}^{-}}+\frac{a_{j+\frac{1}{2}}^{+} a_{j+\frac{1}{2}}^{-}}{a_{j+\frac{1}{2}}^{+}-a_{j+\frac{1}{2}}^{-}}\left[U_{j+\frac{1}{2}}^{+}(t)-U_{j+\frac{1}{2}}^{-}(t)\right]
$$

Compared with classical WENO or WENO-Z reconstructure, we adopted an improved WENO-Z method in terms of higher resolution.

where [11]

$$
u_{j+\frac{1}{2}}^{-}=P_{j}\left(x_{j+\frac{1}{2}}\right)=\sum_{k=0}^{2} w_{\mathrm{k}} u_{j+\frac{1}{2}}^{(k)}, \quad \sum_{k=0}^{2} w_{k}=1
$$




$$
\left.\begin{array}{l}
u_{j+\frac{1}{2}}^{0}=\frac{1}{3} u_{j-2}-\frac{7}{6} u_{j-1}+\frac{11}{6} u_{j} \\
u_{j+\frac{1}{2}}^{1}=-\frac{1}{6} u_{j-2}+\frac{5}{6} u_{j}+\frac{1}{3} u_{j+1} \\
u_{j+\frac{1}{2}}^{2}=\frac{1}{3} u_{j}+\frac{5}{6} u_{j+1}-\frac{1}{6} u_{j+2}
\end{array}\right\}
$$

The weights $w_{k}$ are defined as [12]

$$
w_{k}^{z p}=\frac{\alpha_{k}^{z p}}{\sum_{l=0}^{2} \alpha_{l}^{z p}}, \quad \alpha_{l}^{z p}=d_{k}\left[1+\left(\frac{\tau+\varepsilon}{\beta_{k}+\varepsilon}\right)^{p}+\lambda\left(\frac{\beta_{k}+\varepsilon}{\tau+\varepsilon}\right)\right]
$$

where we set $p=2, \lambda=\Delta x^{2 / 3}$ empirically in our tests. The smoothness indicators $\beta_{k}$ and ideal weights $d_{k}$ can be found in [11].

For the time discretization, we adopt the third order TVD Runge-Kutta method [11]:

$$
\begin{aligned}
& u^{(1)}=u^{[n]}+\Delta t^{[n]} L\left(u^{[n]}, t^{[n]}\right), \\
& u^{(2)}=\frac{3}{4} u^{[n]}+\frac{1}{4} u^{(1)}+\frac{1}{4} \Delta t^{[n]} L\left(u^{(1)}, t^{[n]}+\Delta t^{[n]}\right), \\
& u^{[n+1]}=\frac{1}{3} u^{[n]}+\frac{2}{3} u^{(2)}+\frac{2}{3} \Delta t^{[n]} L\left(u^{(2)}, t^{[n]}+\frac{1}{2} \Delta t^{[n]}\right) .
\end{aligned}
$$

\section{Numerical Experiments}

We considered an example of nine classes of road users studied by Wong and Wong (2002) to illustrate the capability of the MCLWR model in properly describing platoon dispersion. Consider a $2 \mathrm{~km}$ long highway. We adopt the speed-density relationship modified from Drake et al. (1967),

$$
u_{m}=v_{m}(\rho)=u_{\mathrm{f} m} \exp \left(-\left(\rho / \rho_{0}\right)^{2} / 2\right), \quad m=1,2, \ldots, 9,
$$

where $u_{\mathrm{f} m}$ is the free flow speed of user class $m$, and the optimal density $\rho_{0}$ was set to $50 \mathrm{veh} \cdot \mathrm{km}^{-1}$. The distribution for these user classes is presented in Fig.1.

case 1(Non-congested traffic regime) The initial platoon in the non-congested traffic regime is shown in Fig.2. The Fig. 3 gives the computational results of two schemes by using $N=400$ grid points, here the CFL is taken as 0.48 and the output time is $t=0.015 \mathrm{~h}$. The solid line in figures computed by 12800 grid points is considered as the exact solution. As can be seen from Fig.3, our method in this paper have higher resolution, and is superior to the WENO-Z scheme. The Fig. 4 gives the results of total density at different times, we can see the evolution of the platoon dispersion and staircases presence, user classes could overtake and drive at desired rate in non-congested traffic regime, the tail and the font of the platoon has dispersed off gradually with times evolution.

case 2(Congested traffic regime) The initial platoon in the congested traffic regime is shown in Fig.5. The Fig. 6 gives the results of two schemes at $T=0.045 \mathrm{~h}$. The Fig. 7 gives the pictures of total density at different times. It is clear to see that dispersion at the tail of the platoon is limited and ongoing gradually later until its density value has dropped to near or below certain critical values which are the optimal densities. When the platoon has a density less than the optimal density, the traffic regime is non-congested, the procedure of platoon dispersion is similar to that in non-congested traffic regime, while the front of the platoon can disperse gradually with time evolution due to the empty vehicles downstream. 


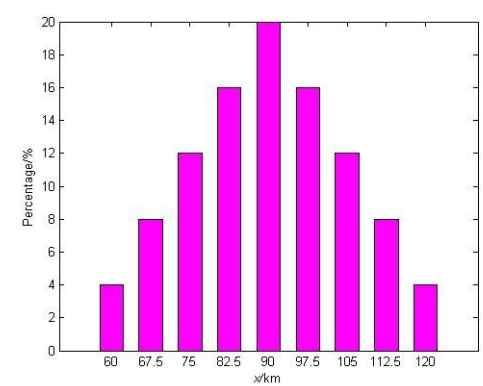

Fig. 1: Platoon dispersion: Distribution of free speed

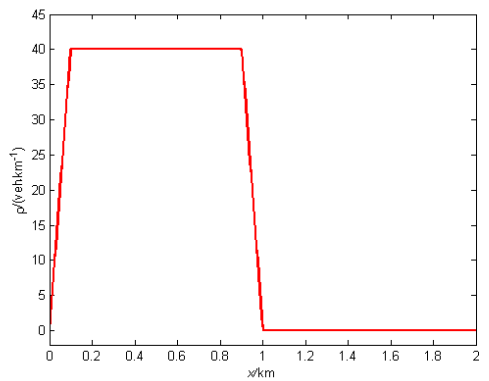

Fig. 2: Non-congested regime: Initial platoon

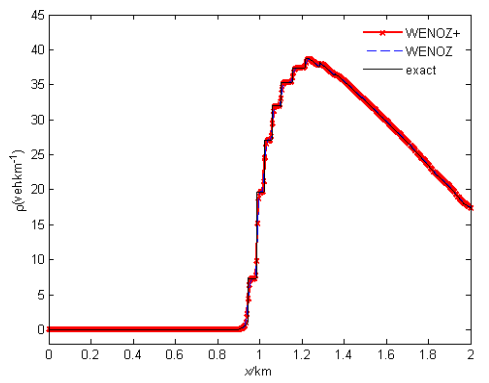

(a) $\mathrm{T}=0.015 \mathrm{~h}$, total density

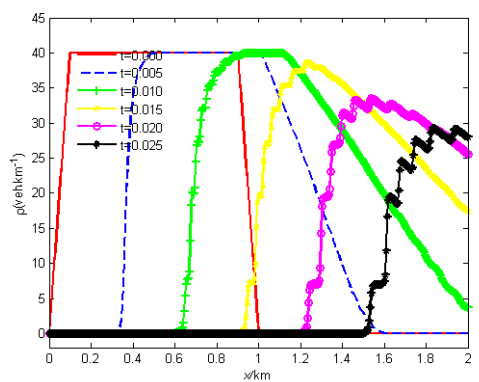

Fig. 4: Non-congested regime: density of various times

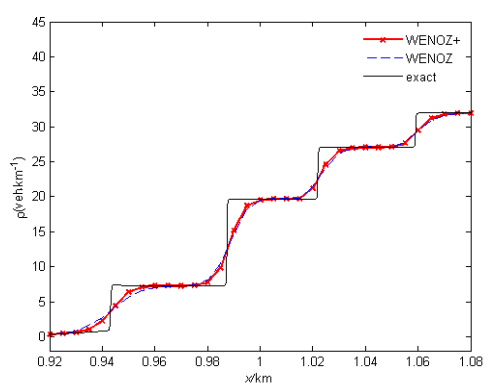

(b) Zoom at $[0.92,1.05]$

Fig. 3: Comparison between WENOZ+ scheme and WENOZ scheme at non-congested state

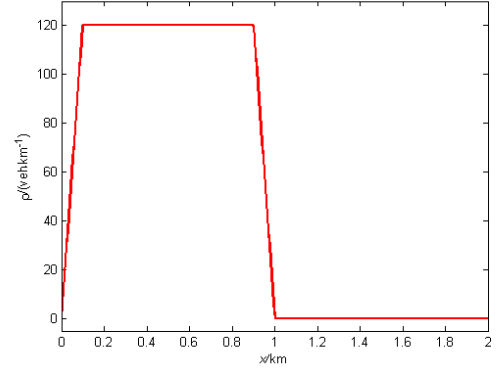

Fig. 5: Congested regime: Initial platoon

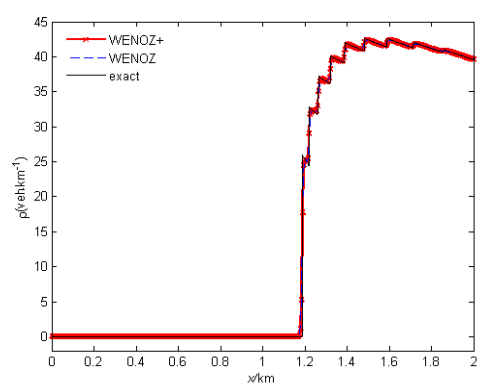

(a) $\mathrm{T}=0.045 \mathrm{~h}$, total density

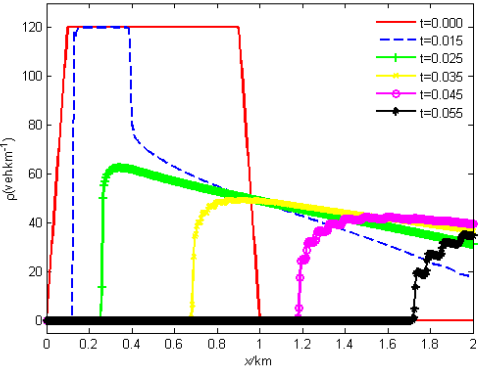

Fig. 7: Congested regime: density of various times

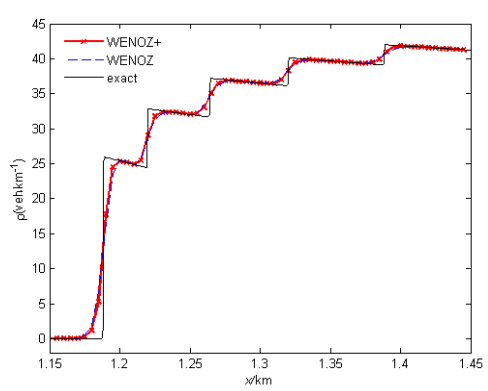

(b) Zoom at $[1.15,1.45]$

Fig. 6: Comparison between WENOZ+ scheme and WENOZ scheme at congested state 


\section{Conclusions}

In this paper, we have derived a high-resolution scheme for the MCLWR model. This new method is based on an improved WENO-Z reconstruction and semi-discrete central-upwind numerical fluxes, and it take full advantages of the simplicity and practicality of SDCU numerical fluxes and the WENO-Z reconstruction with high order of accuracy and non-oscillatory property. Numerical examples show that the method in this paper have higher resolution and less numerical dissipation. In the future, the advanced research on traffic flow problem will have a positive effect on energy and environment field.

\section{Acknowledgements}

This work was financially supported by National Natural Science Foundation of China (91530106, 11571366).

\section{References}

[1] Michael J Lighthill and Gerald Beresford Whitham. On kinematic waves. ii. a theory of traffic flow on long crowded roads. In Proceedings of the Royal Society of London A: Mathematical, Physical and Engineering Sciences, volume 229, pages 317-345. The Royal Society, 1955.

[2] GCK Wong and SC Wong. A multi-class traffic flow model-an extension of LWR model with heterogeneous drivers. Transportation Research Part A: Policy and Practice, 36(9):827-841, 2002.

[3] Mengping Zhang, Chi-Wang Shu, George CK Wong, and SC Wong. A weighted essentially non-oscillatory numerical scheme for a multi-class Lighthill-Whitham-Richards traffic flow model. Journal of Computational Physics, 191(2):639-659, 2003.

[4] Jianzhong Chen, Zhongke Shi, and Yanmei Hu. A relaxation scheme for a multi-class Lighthill-Whitham-Richards traffic flow model. Journal of Zhejiang University SCIENCE A, 10(12):1835-1844, 2009.

[5] D Ngoduy and R Liu. Multiclass first-order simulation model to explain non-linear traffic phenomena. Physica A: Statistical Mechanics and its Applications, 385(2):667-682, 2007.

[6] Alexander Kurganov, Sebastian Noelle, and Guergana Petrova. Semi-discrete central-upwind schemes for hyperbolic conservation laws and Hamilton-Jacobi equations. Siam J.sci.comput, 23(22):707-740, 2000.

[7] Sigal Gottlieb, Chi Wang Shu, and Eitan Tadmor. Strong stability preserving high order time discretization methods. Siam Review, 43(1):89-112, 2000.

[8] Peng Zhang, Ruxun Liu, SC Wong, and Shiqiang Dai. Hyperbolicity and kinematic waves of a class of multi-population partial differential equations. European Journal of Applied Mathematics, 17(2):171-200, 2006.

[9] Peng Zhang, S. C. Wong, and Shi Qiang Dai. A note on the weighted essentially non-oscillatory numerical scheme for a multi-class Lighthill-WhithamRichards traffic flow model. Communications in Numerical Methods in Engineering, 25(11):1120-1126, 2009.

[10] Jianzhong Chen, Yanmei Hu, Jianhu Feng. A semi-discrete central-upwind scheme for multi-class Lighthill-Whitham-Richards traffic flow model (In Chinese). Chinese Journal of Computational Physics, 31(3):323-330, 2014.

[11] Guang Shan Jiang and Chi Wang Shu. Efficient implementation of weighted ENO schemes. Journal of Computational Physics, 126(1):202-228, 1996.

[12] F.Acker, R.B.de R.Borges, B.Costa. An improved WENO-Z scheme[J]. Journal of Computational Physics, 2016, 313:726-753. 